\title{
Implementation and Evaluation of an e-Learning Architecture on Cloud Environments
}

\author{
Chao-Tung Yang, Wei-Ting Yeh, and Wen-Chung Shih
}

\begin{abstract}
In this work, we proposed an e-learning architecture based on cloud computing technologies. Also, we demonstrate how to construct a complete e-learning cloud containing the essential components for deployment stage and provide an example of the experimental application to further analyze its pros and cons against the conventional method. Furthermore, we propose how to provide the ubiquitous e-learning capability from an e-learning cloud. By utilizing a smart mobile application combined with the Quick Response Code mechanism, we can offer the education provider, students and participant the ability to offer and obtain information or knowledge more quickly and easily in many e-learning application environment including schools, museums and exhibitions. This architecture contains a private cloud on local hardware resources and SaaS services powered by the external large-scale cloud services provider.
\end{abstract}

Index Terms-E-learning, cloud computing, virtualization, VM migration, live migration.

\section{INTRODUCTION}

Nowadays, e-learning and Cloud computing are evidently the mainstream and trends while the former in education and the later in information technology, thus the know-how of combining them with advantages are widely presented. The first step is usually concerning the model and the elements of integrated e-learning and Cloud computing [1]-[8]. Next, we will face to transform the e-learning services from conventional platforms to current cloud architecture platforms [9], [10]. Moreover, the barrier and challenge going with pros and cons that should be noticed have been discussed [11], [12]. Meanwhile the increasing development of mobile devices leads to the popular application to e-learning within cloud services. This can be considered as an expanded utilization of integrating e-learning and Cloud computing accompanying with more technique or mechanism, for instances the Quick Response Code and context-awareness [13]-[19]. However, after surveying literatures we will rarely find a case that contains all above subject and unites the whole elements. Thus we do effort to organize all above subject and elements and then propose a well functional e-learning environment on Cloud within good flexibility and scalability. It is also low-cost by utilizing open-source resources.

E-learning [20] was a result of the forth industrial

Manuscript received May 12, 2016; revised July 19, 2016.

Chao-Tung Yang and Wei-Ting Yeh are with the Department of Computer Science, Tunghai University, Taichung, Taiwan, R.O.C. (e-mail: ctyang@thu.edu.tw).

Wen-Chung Shih is with the Department of Computer Science and Information Engineering, Asia University, Taichung, Taiwan, R.O.C. (e-mail: wjshih@asia.edu.tw; Corresponding author). revolution also known as the information revolution which leads to popularization of the personal computers and World Wide Web that allowed the digitalization of education tools and environment. e-learning set itself apart from the traditional mediums by focusing heavily on multimedia presentations with comprehensive knowledge management and search system; therefore, e-learning changed the conventional communication between teachers and students which in turn transformed the very essence of education. Since the immense population growth of the personal computers throughout the 80 s, e-learning had gone through many major improvements over the past 30 years. During the first 10 years, e-learning 1.0 stressed on the proper usage of the internet, then version 2.0 began to utilize web2.0 and further enhance interactive applications; finally the new generation of e-learning underline the importance of socializes learning through social software such as blog, wiki, podcasting, and the virtual 3D reality world of "Second Life." Meanwhile, cloud computing was one of the most significant advancement for information technology in the past decade, and had since became the mainstream trend. With its establish grid computing that introduced numerous layers of services, everyone from academic fields to business, governmental officials to average consumers, was enjoying the revolutionary experience of cloud computing.

By analyzing the characteristics of cloud computing, the advantages it would bring to e-learning became easily apparent. First of all, e-learning developers (e.g. teacher, teaching assistant or trainer) no longer needed to worry about the minor balance between hardware and resources, consequently lower their dependency on professional knowledge. Secondly, users of e-learning systems (e.g. students or workers) would only require having some light weight, low cost devices, such as tablet, ultra book, or smart phone, to interact with the e-learning systems through internet connection. Finally, all data used with the e-learning program whether they were personal notes or progress reports would be saved and backup automatically which greatly reduced data management time the storage spaces with improved security. Base on the above summary, we believed the correlation of cloud computing with e-learning system would take the place of the conventional approach that combined the fields of information technology and education. Hence our work would explain the model created by fusing e-learning environment into cloud computing architecture along with the set up procedures, as well as producing our own e-learning cloud to provide an overview of its advantages comings for future refinement and expansion. The Taiwan NCHC's Ezilla cloud platform approach contained most important factors and developed by integrating stable open-source cloud 
software resources including KVM, Opennebula and MooseFS, plus additional module to provide the rapid deployment capability and well flexibility and scalability, thus we apply this approach as our cloud computing environment's base. We proposed the model contained whole architecture and all elements, the deployment essentials, and context-awareness ubiquitous learning method by design and combine an android APP as the supplement so to take advantage of today's mobile device application trend. We finally conduct experiments as simulation of the reality and measure the results to verify the availability, flexibility and scalability in our e-learning cloud environment approach.

\section{SYSTEM DESIGN AND IMPLEMENTATION}

\section{A. The Model of e-Learning Cloud}

This section describes the creation of a fully functional e-learning Cloud Model and its elements. First, we analyze the traditional e-learning to indicate the various components, including the Digital Educational Materials, the Discussion Requirement, Research Demand, Teaching Function, Search Function, and Professional Knowledge Base. Furthermore, we utilize the widely popular social network, as well as some additional needs, such as multimedia playback and sharing, language translation, and website analysis, etc. Collectively we referred to these as "Educational Source and Requirement." According to Fig. 1, these "Educational Source and Requirement" elements will go through a carefully planned conversion known as "Cloudify", which will import numbers of cloud computing technology and software, then divided into the local components of the provisioning hardware/software environments as well as a fully developed free external service (for related software, refer to previous Background info.) After the "Cloudifying" processes take place the completed e-learning Cloud Model as the Fig. 2 showed will consist the following:

\section{1) Virtual classroom}

Use Moodle as a base to create comprehensive functions for managing digital textbook and provide on-line courses with learning assessment review, which can become a good complement/replacement for traditional teaching methods. Such model allows students to receive a wide range of courses, while educators can easily find the instructions they need. Moreover, instructors can monitor students' base on the statistics of the Moodle platform, then adjustment their own teaching according.

\section{2) On-line knowledge base}

Here we use MediaWiki as foundation, since an e-learning environment must have a good knowledge management system to be used as expertise, query, and super- vision and sharing platform.

\section{3) Internet forum}

Build from phpBB, the Internet Forum is an essential part of the e-learning field, which features a complete on-line discussion function for all users, also able to be embedded in a variety of media to present diverse opinions. In addition, it allows for diff erent levels of users (visitors, students, lecturer, and system administrator) to contact each other online and exchange views.

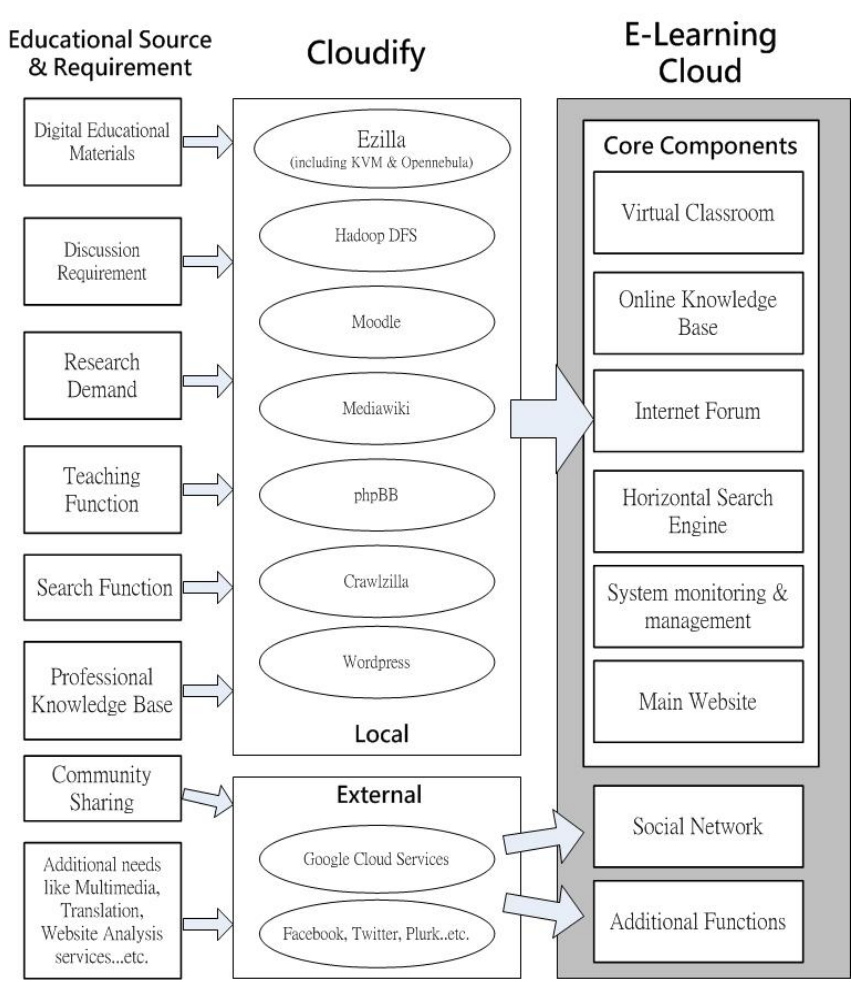

Fig. 1. Core components of e-learning cloud.

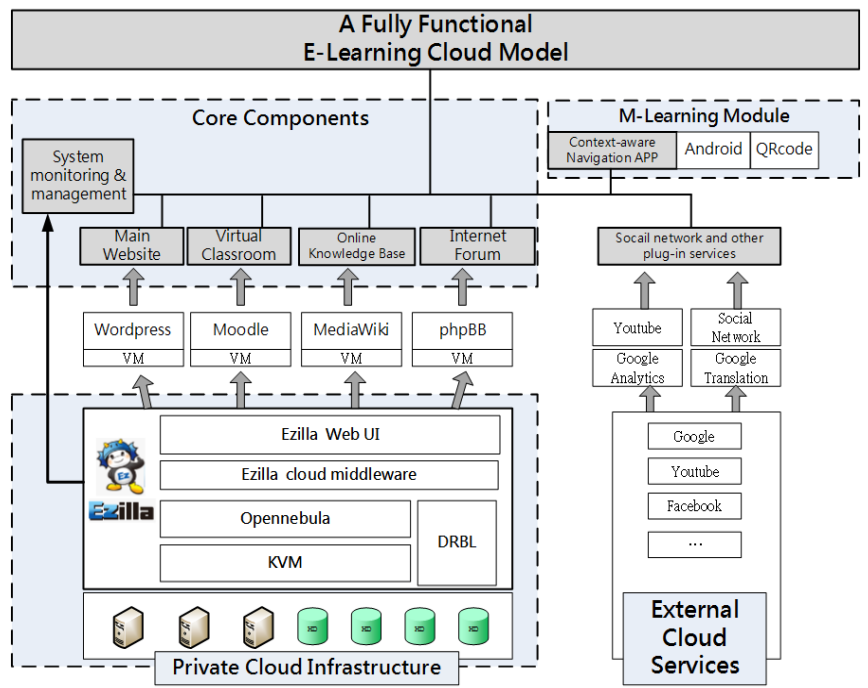

Fig. 2. A fully functional e-learning cloud model.

\section{4) Horizontal search engine}

Horizontal search engine will provide a convenient and comprehensive search feature that allows users to quickly and accurately find any information of e-learning Cloud. We construct this engine using Crawlzilla.

\section{5) System monitoring and management}

This section is related to the IaaS and PaaS parts of cloud computing. We use KVM within Opennebula and Hadoop to construct the virtualization environment and establish management and monitoring mechanisms. This sector will be responsible for managing the whole local infrastructure. It will expand the benefits of IaaS and PaaS cloud computing to the entire e-learning Cloud, which includes the use of Load-balance technique in order to maintain the stability of 
the whole system and reduce energy consumption. As well as using distributed storage and system backup mechanism to prevent accidental data lost and service interruptions. On the other hand, the well design monitoring system will allow IT professionals to better the efficiency of maintenance and adjustment of the underlying hardware resources. Furthermore, PaaS will provide amateur software developers with the most convenient and time efficient platform, while stay mostly invisible to the common e-learning Cloud users.

\section{6) Main website}

A complete e-learning Cloud should have a main site as central hub to connect all other components of the platform, and serve as the entrance of the entire cloud. It should provide an excellent user interface, with easy- access tutorial, so all users can quickly understand the various functions of the e-learning cloud. Here we use Wordpress to build this website.

\section{7) Social network}

Social network is among the most popular of knowledge sharing network, where currently the majority of e-learning services are connected with. A linked social network allows e-learning Cloud to distribute knowledge faster with instant feed- back and greater learning benefit.

\section{8) Additional e-learning functions}

Aside from these core components, the user may also want additional features, such as multimedia playback and sharing, diff erent language translation, external information search, advanced data analysis capabilities, online file sharing/editing, and etc. There are worldwide cloud service providers like Google or Dropbox which provides a lot of well-developed free cloud software services. All these features can be used if necessary when construct our own e-learning Cloud.

\section{B. The Deployment of e-Learning Cloud}

This section we present the Deployment process of our e-learning cloud which will be divided into seven stages:

1) Infrastructure Construction The first stage will apply virtualization technique in cloud computing to all hardware resources to form a re-allocable hardware resources pool. Here we use Taiwan NCHC's Ezilla (including KVM and Opennebula) to create the virtualization environment and the virtual machine's managing functionalities. A combination of KVM plus Opennebula, KVM creates the virtual machine while Opennebula manages the virtual machine's functionalities. In addition, we also use Hadoop to build a distributing file suitable for the massive data storage and handling, as well as a base for horizontal search engine services.

2) Core Components' Base Template Creation This stage will utilize the architecture employed in the previous stag, resulting in a blank virtual machine. Then the installation of the essential software including Moodle, MediaWiki, phpBB and Wordpress, afterwards these virtual machines shall become the base templates. In addition, this is also the stage where system administrators should consider the characteristics of the services provided by e-learning cloud and begin to customize the original virtual machines (modified copy of the virtual machines, while retain the original) in order to meet the future demands of the users .If necessary, kept constant contact with both teaching service providers and users to determine their needs.

3) Use Ezilla‘s Web User Interface to Deploy This stage will make use of the core- function-established virtual machine from last stage to provide e-learning services using Ezilla's web interface (with the selected virtual machine online.) The basic requirements for these services, such as computing power, occupied memory and storage resources, and so on should be considered prior to enter this stage. Once completed, all three core elements of e-learning Cloud (Virtual Classroom, Online Knowledge Base and Internet Forum) should function smoothly.

4) Set the Horizontal Search Engine This stage uses the Hadoop part of the Infrastructure from stage 1) as base along with Crawlzilla to build a horizontal search engine that functions across the entire e-learning Cloud.

5) Organize all Components This phase will use the Wordpress template from stage 2) to establish a Main Website that interlinks with other e-learning Cloud service elements to become a complete e-learning Cloud, while also serve as entrance for the users.

6) Include External Cloud Service and Social Network as Additional e-learning Functions This stage is divided into two parts: I. Apply free application service provided by outside cloud service suppliers in accordance with specific demands and eliminate the cost for research and development; here we have a few examples: Analysis and Translation services provided by Google, YouTube Multimedia ser- vices. II. Link existing community networks, such as Facebook, Twitter, or Plurk in a variety ways, from simply post evaluation to complete embedded discuss functions, or even connects to the community network with applications services.

7) Monitor and Maintain The last stage will exploit the "Monitor" function of Infrastructure from stage 1) to observe the condition of the hardware resource consumption of the entire working platform, as well as the load balance and fault tolerance of the virtualization technology. If a problem occurs, the system administrator should notify IT professionals to make adjustments to the detailed parameters or hardware resource allocation.

After the completion of steps 1) to 7), a fully functional e-learning Cloud is formed with its operational structure shown in Fig. 3.2, which provides a complete e-learning service. In this case, the computing resources' are primarily used by the hardware resources of the local infrastructure, as well as the application services supplied by external cloud service providers.

\section{Ubiquitous e-Learning Cloud}

QR code Application By importing the QR code the whole e-learning cloud system gains the ability that education provider could easily use to provide the environment learning navigation function. The Quick Response Code is a welldeveloped barcode system which has high information capacity and flexibility that can easily be created by the educator or accessed by the participator and learner. 
Mobile Application (Mobile APP) Mobile APP is the part of our architecture' s core components to provide mobility. By utilizing the APP obeyed our design users can scan the QR code tag through mobile devices' camera so to access the learning information, and offer the exhibition guidance function. Furthermore the APP can record the learning information data (including name, links) from the QR code into the e-learning portfolio and afterward uploads the portfolio to the education cloud so the learner can re-access the exhibition items' information and experiences later.

E-portfolio Module E-portfolio module is another core component that gather the statistics and summarize the exhibition's information and learner's experience, creating the environment for learners to regain the exhibition information and experience. Furthermore, the Context-Aware Exhibition Navigation Module Exhibition navigation module provides the learners the concept of situational choices. Learners can choose from a variety of different contexts designed by education providers to participate. This module requires the combine effort of Education provider and IT supporters to formulate the important content of every single context configuration file (txt file), so that once the desired context is selected, it can be downloaded by the mobile app from education cloud. The main contents and structure of the context will be organized in the form of exhibit No. for navigation. Example: Context $[\mathrm{A}]: 1 \rightarrow 3 \rightarrow 5 \rightarrow 26 \rightarrow \ldots$

\section{Integrating the m-Learning Capability into e-Learning Cloud Model}

Fig. 3 presents the whole Context-aware Exhibition Navigation System's Architecture and operation relationship. There are three roles participated in: IT Supporter, Education Provider and Learner (exhibition visitor) IT Supporter takes the responsibility on construction and maintaining of the Education Cloud and the mobile APP, and furthermore need develop the specifications of the con- text configuration file and the e-portfolio file with the education provider. The Education Provider and Learners' job and behavior will be described afterwards.

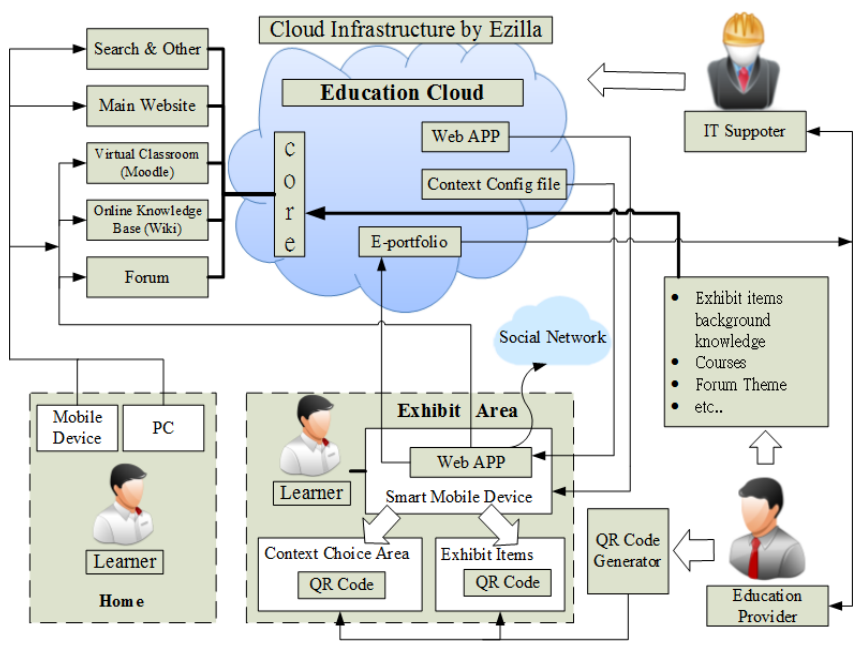

Fig. 3. The architecture of context awareness exhibition navigation system.

\section{1) Android APP's operational processes of design elements}

QR Code reading ability By calling the external QR Code software or including the QR code open source program package from Github on our Android APP to analyze QR code for related Exhibits and gain the corresponding web link (URL link) to the Education cloud for access the knowledge.

Context choice module Reading the context choice section of the QR Code at the front of the exhibition area to download the appropriate context configuration file from the Education cloud and get navigation information with organized exhibition items' number in proper order then send to Navigation module.

Navigation module Use Context choice module's information guide line to rearrange exhibits in specific orders for every situation. Additionally module will store a viewing record to remind learner on whether the exhibits have been covered.

Achievement module Achievement module checks to see if all the corresponding exhibit QR codes in Context have been scanned by the learner then display the accomplishment status. This is a module that can be used to encourage users to complete the education providers' design course.

E-Portfolio module E-Portfolio module will record a numbers of learners progress, including:

1) Name of exhibition

2) Context downloaded

3) Context completion rate and time

4) Corresponding URL link in Education cloud knowledge from all of the exhibits scanned

5) Total time spent

An exhibition Portfolio file is created after each completion and will prompt learners to rename the file base on proper format and learner code to upload it to Education cloud or the appropriate free cloud space

APP Reset module This module will wipe all previous information to reset the APP back to the original state. Moreover, before the implementation of such action the module will automatically output a portfolio file as backup to prevent accidental progression lost.

For education providers, the proposed system creates pamphlets and teaching materials based on exhibition that will be saved onto education cloud's wiki (Online Knowledge Base) and Moodle (Virtual Classroom). It also provides detailed knowledge of the exhibits introduced while Moodle supplies the relevant courses and video educational experiences. Furthermore, the forum section of education cloud will off er discussions in related topic, so that learners and educators can gain feedback from appropriate sources.

\section{2) Android exhibition navigation APP's design and} activity

As shown in Fig. 4, user's(learner) interaction process with our exhibition navigation APP and the APP's operating mechanism with our e-learning cloud can be summarized and listed in 5 stages.

First, use exhibition DM or QR Code link at the entrance to download and install the mobile app from the education cloud to the mobile device.

Browsing through summaries of scenarios in the selection area and use the app to scan QR Code of whichever section that catches one's interest to get connected to the Education cloud's context configuration file. The app will then display 
the concept development flowchart that corresponding to exhibition process numbers, for example: Context [A]: 1>3> 5> 26>....

When the visiting each, simply scan the proper QR Code, and the app will automatically show to the corresponding information and materials associated with the exhibits from the Education cloud.

The APP's e-portfolio module will record individual's learning progression (including user-selected scenarios and practical concept development process, which may be different), and all the QR Code information that were scanned. Also a text file documenting the learning succession will be created which the APP will recommend users to upload to the Education cloud as reference for education providers.

With the APP's recording, the learner will be prompted the learner about what have been seen, and store the info as learning history. Education providers can also use these records to design a fun and interesting reward system similar to collect travel stamps, or game token which may then be exchanged for gifts or souvenirs.

\section{EXPERIMENTAL RESULTS}

In this work, the experiments focus on the two dimensions. First we ensure the whole e-learning system can be deployed on the Ezilla platform, and compare the time spent with traditional environment. Second we test our system's flexibility and extensibility by utilizing the migration and live migration function in Ezilla. The migration mechanism can transfer the e-learning service module on virtual machine from one computing host to another host, thus offering the ability to reallocate the computing resources for services. Moreover, the advanced migration type called Live Migration can further afford the capability that won't cause the service of e-learning service module to stop for a long time, so we don't have to stop the e-learning cloud service when we add new computing host to the infrastructure and then migrate one e-learning service module to the new host. Since the Ezilla platform has two different operation mode: Diskless and Disk, each has different feature, pros and cons, thus our experiment will run separately on two modes, and compare and analyze the results.

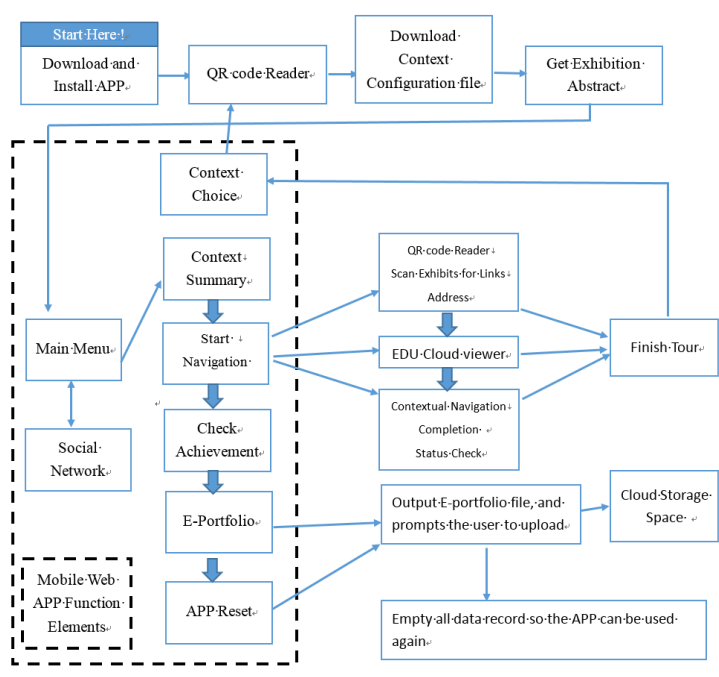

Fig. 4. Learner's interaction process with the navigation APP.

\section{A. Experimental Environments}

As shown in Table I, Our Ezilla Diskless experimental environment has one master node and 4 slave nodes, totally 18 logic cores and 44GB ram for computing resources. Only the master node has 30GB SSD disk space, all with Ubuntu12.04 OS and Gigabit network.

TABLE I: EZILLA DISKLESS ENVIRONMENT HARDWARE SPECIFICATION

\begin{tabular}{llll}
\hline \hline Node & CPU & Memory & Software \\
\hline Master & $\begin{array}{l}4 \text { vcores } \\
(\mathrm{i} 7-2630 \mathrm{QM})\end{array}$ & $4 \mathrm{~GB}$ & $\begin{array}{l}\text { EZilla Master and } \\
\text { Front End in } \\
\text { VMware upon } \\
\text { Win8.1 }\end{array}$ \\
& & & \\
Slave 1 & Q9550 2.83GHz & $8 \mathrm{~GB}$ & EZilla Computing \\
Slave 2 & Q9550 2.83GHz & $4 \mathrm{~GB}$ & Node (Slave Node) \\
Slave 3 & G540 2.5GHz & $16 \mathrm{~GB}$ & \\
Slave 4 & I7-4770K 3.5GHz & $16 \mathrm{~GB}$ & \\
\hline \hline
\end{tabular}

As shown in Table II, Our Ezilla Disk experimental environment has one master node and 4 slave node, 1 vm node as moosefs client, with totally 18 logic cores, 44GB ram and 3536GB disk space for computing resources, all with CentOS6.4 OS and Gigabit network.

As shown in Fig. 5, Slave Node04's CPU Intel I7-4770K has the overwhelming computing power, if we set the performance bench on $17-4770 \mathrm{~K}$ to 10 , then the Intel Q9550 is 4, at last the Intel Celeron G540 is 2. The data is based on Passmark Software's benchmark database.

TABLE II: EZILLA DISK ENVIRONMENT HARDWARE SPECIFICATION

\begin{tabular}{|c|c|c|c|}
\hline Node & CPU & Memory & Software \\
\hline Master & $\begin{array}{l}4 \text { vcores } \\
(\mathrm{i} 7-2630 \mathrm{QM})\end{array}$ & 4GB & $\begin{array}{l}\text { EZilla Master and Front } \\
\text { End in VMware upon } \\
\text { Win8.1 } \\
\text { MooseFS Master and } \\
\text { Client }\end{array}$ \\
\hline $\begin{array}{l}\text { Moose } \\
\text { Client }\end{array}$ & $\begin{array}{l}2 \text { vcores } \\
\text { (i7-2630QM) }\end{array}$ & $2 \mathrm{~GB}$ & $\begin{array}{l}\text { MooseFS Client on } \\
\text { VMware upon Win8.1 }\end{array}$ \\
\hline Slave 1 & Q9550 2.83GHz & $8 \mathrm{~GB}$ & \\
\hline Slave 2 & Q9550 2.83GHz & $4 \mathrm{~GB}$ & EZilla Computing Node \\
\hline Slave 3 & $\mathrm{G} 5402.5 \mathrm{GHz}$ & $16 \mathrm{~GB}$ & (Slave Node) \\
\hline Slave 4 & $\mathrm{I} 7-4770 \mathrm{~K} 3.5 \mathrm{GHz}$ & $16 \mathrm{~GB}$ & \\
\hline
\end{tabular}

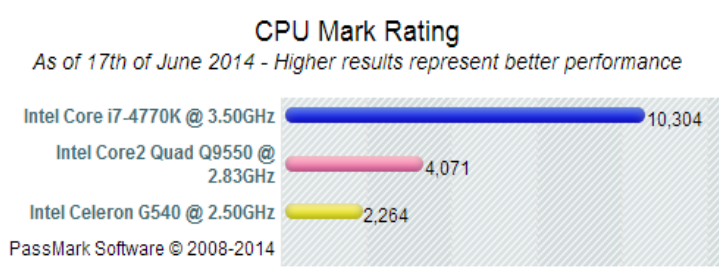

Fig. 5. Learner's interaction process with the navigation APP.

\section{B. Experiment 1: The e-Learning Service Modules Deploy Time Comparison}

In this section, we compare the time cost between 3 infrastructure, and calculate the results. The e-learning service module include 4 core components: Wordpress, Moodle, MediaWiKi, PhpBB. First we compare the deployment time, including the base OS installation and the e-learning service module on bare metal and VM from Ezilla.

And then we use the e-learning service module installed hard disk drive(HDD) image as the default HDD image, and 
setup the template on Ezilla (CPU:2 vcore, Ram: 2GB), then we use the same template to deploy VM on different host with different CPU and compare the VM deployment time.

TABLE III: The INITIAL E-LeARning SERVICE Module DePloyment Time

\begin{tabular}{lll}
\hline \hline & $\begin{array}{l}\text { Deployment } \\
\text { Time }\end{array}$ & Hardware Spec. \\
\hline Bare Metal & 42 min & $\begin{array}{l}\text { CPU: i7-4770K 8core, 8GB } \\
\text { RAM, 120GB SSD }\end{array}$ \\
$\begin{array}{l}\text { VM from EZilla } \\
\text { Diskless }\end{array}$ & $50 \mathrm{~min}$ & VCPU: i7-4770K 4core, 4GB \\
VM from EZilla Disk & $65 \mathrm{~min}$ & RAM, 12GB SSD \\
\hline \hline
\end{tabular}

TABLE IV: THE VM CONTAINED E-LEARNING SERVICE MODULE DEPLOY TIME WITHIN DIff ERENT CPU MODEL

\begin{tabular}{lll}
\hline \hline Host & $\begin{array}{l}\text { EZilla Diskless } \\
\text { Deployment Time }\end{array}$ & VM Template \\
\hline i7-4770K & 33s to 6s & CPU: 2 vcore, 2GB RAM \\
Q9550 & 21 s to 8s & CPU: 2 vcore, 2GB RAM \\
G540 & 31 s to 6s & CPU: 2 vcore, 2GB RAM \\
\hline \hline
\end{tabular}

\section{Experiment 2: The Migration and Live Migration Mechanism Performance Comparison}

In this part, we first present the measurement the time spent on migration and live migration in Ezilla Diskless and Ezilla Disk. As shown in Fig. 6 and 7, the VM migration mechanism costs more time in Ezilla Diskless edition. The live migration mechanism spends less time than cold migration mechanism, and get a clear improvement time cost under Ezilla Disk environment.

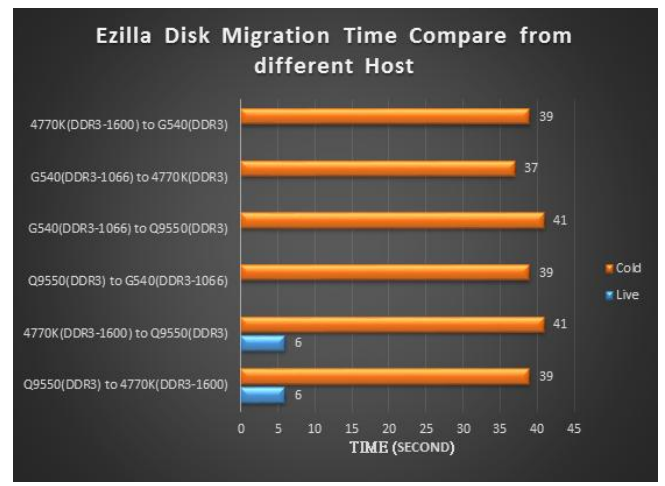

Fig. 6. Ezilla diskless migration time comparison from different host.

Furthermore, we utilize tools called JMeter and Badboy to create a simulation environment that multiple people accesses our e-learning service cloud. First Badboy can record user's interaction with web-based service and then output a script for JMeter. We use JMeter to import this script from Badboy as one user's reaction for once, and we adjust JMeter's parameter configure to create a multiple-people access simulation situation and so to stress our e-learning service cloud. Now we can experiment if our platform is under the real internet environment and we need to migrate the VM bearing the e-learning service module cause we want to adjust and reallocate our infrastructure under different service loading. A thread can count as one user requesting access to the e-learning service, and the ramp-on period 20 s means all users will send request in 20 seconds. The loop counter 10 means repeat the situation 10 times, at last the constant delay offset $5000 \mathrm{~ms}$ with random delay maximum $2000 \mathrm{~ms}$ means every user will spend 57 seconds on a webpage and then forward to the next one. All test environment are on the VM with OS ubuntu12.04, 2 core vcpu and 2GB ram, we always execute the life migration process from host with Q9550 to host with $4770 \mathrm{~K}$ while on JMeter stressing.

We can see that performed live migration with Ezilla Disk result in faster completion time, lower system downtime, and smaller impact to user who access our e-learning cloud services platform, as well as less information obtained response time than the Ezilla Diskless. In addition, observing the time differences between the point of distribution and divergence on the map, you can also get information on the system at the time of Ezilla Disk during heavy usage with relatively low response time and high stability, clearly benefited from the higher I/O performance. However, live migration was not flawless, we conclude that when the data write-in rate, caused by many user' $s$ request to the e-learning cloud service on VM, surge to close or even exceeds, the data transfer and replication rate to different host' s memory from the migration mechanism, the whole migration process will be greatly delay where it will have to wait for network data transfer request generated by users to reduce, which significantly extending the data access and response time from e-learning cloud services, and even temporarily interrupt user access to the e-learning services.

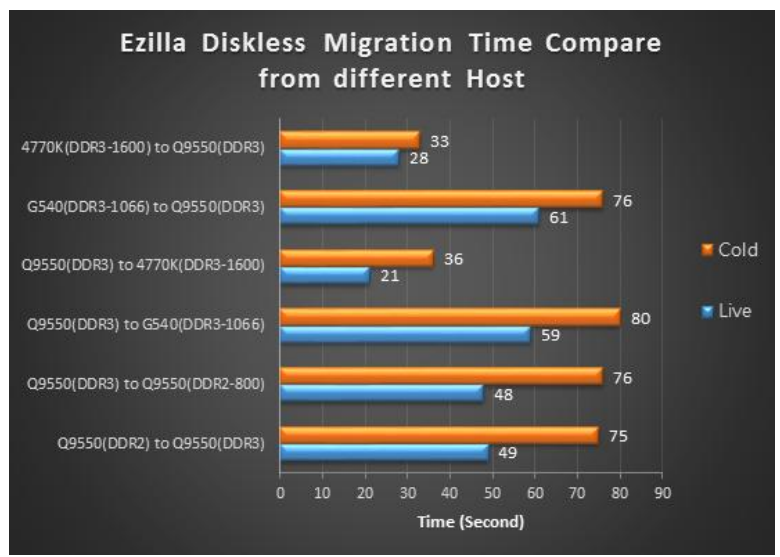

Fig. 7. Ezilla disk migration time comparison from different host.

After all, this experiment proved the e-learning environment on Ezilla cloud we proposed have better system expandability and availability than traditional methods without cloud computing technique, because we can add more computing resources more quickly and easily, while don't need to stop the e-learning services during upgrading. The Ezilla Diskless solution has less performance during heavy loading but less cost and more easy to deploy, in contrast the Ezilla Disk solution has more loading capacity and performance but also need more cost spent on hard disks.

\section{Combining the Exhibition Navigation APP and the THU K-16 Education Cloud}

As shown in Fig. 8, first we start the Exhibition Navigation APP and can get the Exhibition Introduction from our THU-K16 Education Cloud. Next click the "Get Navigation Context Button" on the right downside to scan the exhibition context QR code to acquire the context data. Then, we can see the navigation context show on the lower half, and change to the order by exhibition item number. So we click the button on the top to scan the first exhibition item number. The 
No.001 exhibition item's relevant information on our e-learning cloud is shown on the screen, also with its order number beneath.

\section{CONCLUSION}

Nowadays, the demand for e-learning is constantly increasing. However, the escalating expenses to create such teaching and learning environments also becomes a major issue which every educational organization must face. Furthermore, there are other difficulties, including the development of educational software and the underlying construction of hardware environments; fortunately, the popularization for open source materials along with the continuous advancement of cloud computing technology greatly reducing the cost and technical threshold of the e-learning environment.

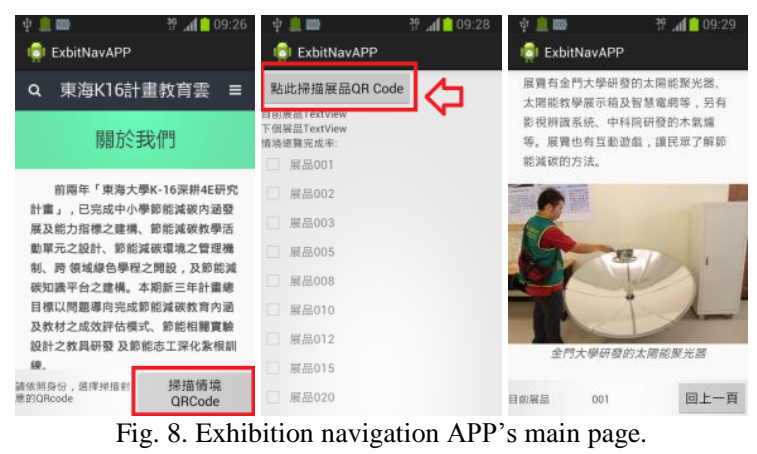

This work presents a complete solution through the integration of e-learning, cloud computing, open source software, and external cloud resources to provide deployment method for more learners and educators. In the end, the work proposed through a combination of e-learning, cloud computing and open source spirit, to indicate the key elements of a complete e-learning cloud, and to help expanding e-learning education throughout the world. In addition, we have assembled a Mobile APP with E-portfolio, Context-awareness mechanism and QR code amounts other elements to create, through detailed planning, a low-cost, easy to access the e-learning cloud which is ubiquitous in hope to bring education providers and learners a better education experience. In order to achieve the goal of low cost and easy to use, we purposely avoid the more complex and expensive indoor navigation technology (systems that requires $\mathrm{Wi}-\mathrm{Fi}$ or Bluetooth technique to provide accurate position capability), our system is designed to be promoted to with limited budget and manpower while providing the best education ubiquitous cloud service experience.

\section{ACKNOWLEDGMENT}

This research was supported by Ministry of Science and Technology of Republic of China under the number of MOST 104-2511-S-468-004 and MOST 104-2511-S-468 -001 -MY2.

\section{REFERENCES}

[1] I. Carol, G. Roy, and A. Prassanna, "A cloud model for effective e-learning," Computing and Communication Technologies (WCCCT), pp. 167-169, Feb. 2014.
[2] F. Doelitzscher, A. Sulistio, C. Reich, H. Kuijs, and D. Wolf, "Private cloud for collaboration and e-learning services: from iaas to saas," presented at Computing, 2011.

[3] A. Fernández, D. Peralta, F. Herrera, and J. M. Benítez, "An overview of e-learning in cloud computing," Workshop on Learning Technology for Education in Cloud (LTEC'12), pp. 35-46, 2012.

[4] R. Hammad, M. Odeh, and Z. Khan, "Towards a generic requirements model for hybrid and cloud-based e-learning systems," Cloud Computing Technology and Science (CloudCom), pp. 106-111, Dec. 2013.

[5] X. Laisheng and W. Zhengxia, "Cloud computing: A new business paradigm for e-learning," Measuring Technology and Mechatronics Automation (ICMTMA), pp. 716-719, Jan. 2011

[6] H. Mousannif, I. Khalil, and G. Kotsis, "Collaborative learning in the clouds," Information Systems Frontiers, vol. 15, no. 2, pp. 159-165, 2013.

[7] K. Verma and M. Rizvi, "Impact of cloud on e-learning," Computational Intelligence and Communication Networks (CICN), pp. 480-485, Sept. 2013.

[8] S. Yina, Y. Fuxing, and S. Dingli, "Campus e-learning system combine with the cloud computing," Electric Information and Control Engineering (ICEICE), pp. 4038-4040, April 2011.

[9] W.-E. Chen, "The experience of using e-learning platform on cloud," The 2nd International Workshop on Learning Technology for Education in Cloud, Springer Proceedings in Complexity, pp. 175-179, 2014.

[10] P. Pocatilu, F. Alecu, and M. Vetrici, "Using cloud computing for e-learning systems," in Proc. the 8th WSEAS International Conference on Data Networks, Communications, Computers, DNCOCO'09, pp. 54-59, 2009.

[11] S. Misra et al., "Acceptance and use of e-learning based on cloud computing: The role of consumer innovativeness," Computational Science and Its Applications (ICCSA 2014), pp. 159-174. 2014.

[12] P. Pocatilu, F. Alecu, and M. Vetrici, "Measuring the efficiency of cloud computing for e-learning systems," Trans. on Comp., vol. 9, no. 1, pp. 42-51, Jan. 2010.

[13] R. S. Cobcroft, S. J. Towers, J. E. Smith, and A. Bruns, "Mobile learning in review: Opportunities and challenges for learners, teachers, and institutions," Online Learning and Teaching (OLT) Conference, pp. 21-30, 2006.

[14] M. Derntl and K. Hummel, "Modeling context-aware e-learning scenarios," Pervasive Computing and Communications Workshops, pp. 337-342, March 2005.

[15] S. Huang and H. Yin, "A new mobile learning platform based on mobile cloud computing," Advances in Future Computer and Control Systems, pp. 393-398. 2012.

[16] M.-Y. Luo and S.-W. Lin, "From monolithic systems to a federated e-learning cloud system," Cloud Engineering (IC2E), pp. 156-165, March 2013.

[17] Y. Mehdipour and H. Zerehkafi, "Mobile learning for education: Benefits and challenges," International Journal of Computational Engineering Research, vol. 3, no. 6, pp. 93-101, 2013.

[18] K. Peters, "M-learning: Positioning educators for a mobile, connected future," The International Review of Research in Open and Distance Learning, vol. 8, no. 2, 2007.

[19] A. Schepman, P. Rodway, C. Beattie, and J. Lambert, "An observational study of undergraduate students' adoption of (mobile) note-taking software," Comput. Hum. Behav., vol. 28, no. 2, pp. 308-317, Mar. 2012.

[20] E-learning. (2014). [Online]. Available: http://en.wikipedia.org/wiki/e-learning

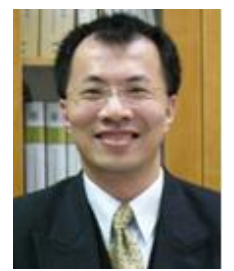

Chao-Tung Yang is a distinguished professor of Computer Science at Tunghai University in Taiwan. He received the $\mathrm{PhD}$ in computer science from National Chiao Tung University in July 1996. In August 2001, he joined the Faculty of the Department of Computer Science at Tunghai University. He is serving in a number of journal editorial boards, International Journal of Communication Systems (SCI, EI) Area Editor, International Journal of Advanced Computer Science and Applications (EI) Associate Editor, KSII Transactions on Internet and Information Systems (SCI, EI), Journal of Applied Mathematics, Journal of Cloud Computing, International Journal of Grid and High Performance Computing (EI), International Journal of Cloud Computing and Services Science (IJ-CLOSER), Journal of Convergence Information Technology(EI), International Journal of Digital Content Technology and its Applications (EI) "Grid Computing, Applications and Technology" Special Issue of Journal of 
Supercomputing, and "Grid and Cloud Computing" Special Issue of International Journal of Ad Hoc and Ubiquitous Computing. Dr. Yang has published more than 280 papers in journals, book chapters and conference proceedings. His present research interests are in cloud computing, big data, parallel computing, and multicore systems. He is a member of the IEEE Computer Society and ACM.

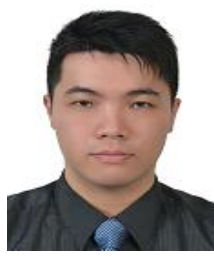

Wei-Ting Yeh received the master degree in compute science at Tunghai University in 2014. His research interests include e-learning, cloud computing, and multicore systems.

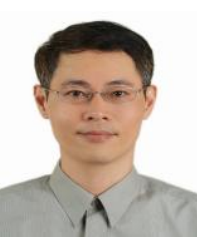

Wen-Chung Shih received the Ph.D. degree in computer science from the National Chiao Tung University in 2007. From 2007 to 2014, he has been on the Faculty of the Department of Applied Informatics and Multimedia at Asia University. From August 2014 he has been on the Faculty of the Department of Computer Science and Information Engineering at Asia University, Taiwan, and is currently an associate professor and chairman there. His research interests include e-learning, cloud computing, expert systems and big data analytics. 